\title{
Differential expression of two novel odorant receptors in the locust (Locusta migratoria)
}

\author{
Haozhi Xu', Mei Guo', Ying Yang ${ }^{1,2}$, Yinwei You ${ }^{1,3}$ and Long Zhang ${ }^{1 *}$
}

\begin{abstract}
Background: Olfaction in animals is important for host localization, mating and reproduction in heterogeneous chemical environments. Studying the molecular basis of olfactory receptor neurons (ORNs) systems can elucidate the evolution of olfaction and associated behaviours. Odorant receptors (ORs) in insects have been identified, particularly in the holometabolous model Drosophila, and some of them have been functionally studied. However, ORs in the locust - a hemimetabolous model insect and the most important insect crop pest-have not yet been identified, hindering our understanding of locust olfaction. Here, we report for the first time four putative ORs in Locusta migratoria: LmigOR1, LmigOR2, LmigOR3 and LmigOR4.

Results: These four putative OR genes encoded proteins with amino acids of 478, 436, 413 and 403 respectively. Sequence identity among them ranged from $19.7 \%$ to $35.4 \%$. All ORs were tissue-specifically expressed in olfactory organs, without sex-biased characteristics. However, LmigOR1, LmigOR3 and LmigOR4 were only expressed in the antenna, while LmigOR2 could also be detected in mouthparts. In situ hybridization demonstrated that the LmigOR1antisense probe labelled olfactory receptor neurons (ORNs) in almost all segments of the antenna, but only a few segments housed ORNs expressing LmigOR2. The number of neurons labelled by LmigOR1 antisense probes in each antennal segment was much greater ( $>10$ neurons/segment) than that labelled by LmigOR2 probes (generally 1-3 neurons/segment). Furthermore, some of the labelled neurons could be attributed to the basiconic sensilla, but LmigOR1 and LmigOR2 were expressed in different subtypes.
\end{abstract}

Conclusions: Our results strongly suggested that these newly discovered genes encode locust ORs and the differential expression patterns of LmigOR1 and LmigOR2 implied distinct functions. These results may offer insights into locust olfaction and contribute to the understanding of the evolution of insect chemoreception.

Keywords: Odorant receptor, Locust, Odorant receptor neuron, Basiconic sensillum, Expression

\section{Background}

Mammals and insects have adapted evolutionarily to the heterogeneous chemical environments in which they live. Odorant receptors (ORs) in ORNs systems are involved in scent detection and discrimination and are therefore key to understanding the molecular evolution of olfactory mechanisms in animals [1-4].

Insect ORs are evolutionarily unrelated to their vertebrate counterparts. Although insect ORs possess seven transmembrane domains like the G-protein-coupled ORs in vertebrates, the transmembrane topology is completely inverted [5-7]. Since the discovery of the first OR

\footnotetext{
* Correspondence: locust@cau.edu.cn

'Department of Entomology, China Agricultural University, Beijing 100193, P. R. China

Full list of author information is available at the end of the article
}

in Drosophila through bioinformatics analysis of the partially sequenced genome $[2,8,9]$, numerous OR-coding genes have been identified in various holometabolous insects. In the genomes characterized to date, 60 OR genes have been found in Drosophila [10,11], 79 in mosquito [11], 162 in honey bee [12], and 341 in red floor beetle [13]. Insect ORs evolve rapidly, and there is considerable sequence diversity among OR proteins-many show only $20 \%$ similarity [14]. The olfactory systems of a variety of insect species have been extensively studied. However, it is still very difficult to draw satisfying conclusions about the evolution of insect olfaction because of the absence of studies on some important taxa, such as orthopteran insects [15].

Most insect ORs are only expressed in olfactory organs such as antennae or maxillary palps [16-19]. Aside from the highly conserved odorant receptor co-receptor (ORco) 
subfamily, the expression of each individual OR is confined to a unique subset of ORNs, resulting in molecular diversity among ORNs. The "One-ReceptorOne-Neuron" model proposed for mammalian olfactory systems also applies to most insect ORNs [2,20]. ORNs expressing the same ORs were housed in electrophysiologically identical sensilla subtypes and converged to the same glomerulus in the antennal lobe. Extracellular single-unit recordings from individual olfactory sensilla have revealed that different odorants elicit responses from different subsets of ORNs, and that ORNs exhibit a remarkable diversity of response properties $[3,4,21]$. ORNs housed in different sensilla types expressed distinct ORs, allowing the sensilla to be characterized by their molecular and cellular properties [2,4,19,21-23].

Locust (Locusta migratoria) is regarded as a model animal of hemimetabolous insects, and is a notorious worldwide pest that has historically caused tremendous damage to agricultural production [24]. Its behaviours, such as feeding, migration, mating, defence, aggregation, and reproduction, are probably mediated by chemoreception. The development of alternative control methods to replace chemical pesticides will depend on understanding the molecular mechanisms that regulate locust behaviour.

Thus far, we have identified and characterized several locust odorant-binding proteins (OBPs) and chemosensory proteins (CSPs) that are thought to be involved in chemoreception in insects [25-29]. Recently, the evolutionally conserved ORco was identified in locust and found to be ubiquitously expressed in ORNs, just as in other insects [30]. However, extensive efforts to identify functionally specific ORs have failed because of the low sequence homology between ORs and the large evolutionary distances among insect clades. The absence of knowledge about ORs that transduce the binding of odorants into neural activity has hindered further understanding of locust olfaction.

Here, we have identified four novel OR-coding genes in locust and found that LmigOR1 and LmigOR2 showed differential expression patterns in olfactory organs. LmigOR1 was specifically expressed in antennae, whereas LmigOR2 transcripts could also be detected in mouthparts. Some of the ORNs expressing LmigOR1 or LmigOR2 could be found in the basiconic sensilla, but the receptors were present in different sensilla subtypes. These results may offer insights into locust olfaction and contribute to the understanding of the evolution of insect chemoreception.

\section{Results}

\section{Identification of odorant receptors in locust}

A tBlastn search of the locust antenna expressed sequence tag (EST) database, using previously identified insect ORs as queries, identified 2 sequence fragments coding putative ORs. Full cDNA length was obtained using RACE.
Iterative blast using the newly identified ORs as query sequences identified another two putative ORs fragments and their full length was obtained by walking sequencing of the clones. The complete coding sequences were deposited in GenBank and designated as LmigOR1 [Genebank: JQ766965], LmigOR2 [Genebank: JQ766966], LmigOR3 [Genebank: KC689310] and LmigOR4 [Genebank: KC689311]. They consisted of amino acids of 478, 436, 413 and 403. The theoretical molecular weights were 50.762, $47.867,44.321$ and $44.805 \mathrm{KD}$ respectively. Sequence identities among them ranged from $19.7 \%$ to $35.4 \%$ (Table 1). A Pfam search clearly classified them into the seventransmembrane (7-TM) superfamily, although TMHMM prediction gave relatively low probabilities for the seventh transmembrane domains, perhaps in part because of ambiguities in the hydrophobic regions. The predicted locations of putative transmembrane domains within the sequences are indicated in Figure 1. Phylogenetic analysis of several more closely related insect ORs confidently grouped LmigORs into a monophyletic lineage, indicating they can be as designated as locust-specific ORs (Figure 2).

\section{The temporal and spatial expression profile of LmigORs}

To assess the tissue-specific expression patterns of the ORs in Locusta migratoria, polymerase chain reaction (PCR) experiments were performed using sequencespecific primers that amplified $\sim 700$-bp sequences from cDNA pools produced from $1 \mu \mathrm{g}$ of total RNA. The LmigOR1, LmigOR3 and LmigOR4 mRNA were detected exclusively in locust antennae, whereas the LmigOR2 transcripts were also abundantly detected in mouthparts. In non-olfactory organs, such as tarsi, wings and guts, we did not detect any specific expression, although numerous gustation-related chemosensilla (chaetic sensilla) were present. We found no differences in tissue distribution between sexes (Figure 3a). Interestingly, we found that the expression levels of LmigOR1 and LmigOR2 in the antennae increased with age, especially after the fourth instar stage. In contrast, LmigOR4 expressed more highly in nymph than in adult. Among these genes, only LmigOR3 could be detected in eggs besides its abundant expression from nymph to adult (Figure $3 b$ ). The locust actin gene was constitutively expressed at high levels in all tissues at all developmental stages, providing a control for the integrity of the cDNA template (Figure 3).

Table 1 Amino acids identities among the four putative locust ORs

\begin{tabular}{lllll}
\hline & LmigOR1 & LmigOR2 & LmigOR3 & LmigOR4 \\
\hline LmigOR1 & $100 \%$ & & & \\
\hline LmigOR2 & $19.6 \%$ & $100 \%$ & & \\
\hline LmigOR3 & $21.7 \%$ & $19.9 \%$ & $100 \%$ & \\
\hline LmigOR4 & $35.4 \%$ & $20.7 \%$ & $25.3 \%$ & $100 \%$ \\
\hline
\end{tabular}




\begin{tabular}{|c|c|c|}
\hline ImigoR1 & . RC्रRIYFIE్FGTVCTSNIHI... GAIAIIRS & 84 \\
\hline ImigOR2 & $\therefore$ CGQGITPEAVVAAATIAAVAF I FAGVVIRL & 60 \\
\hline ImigoR3 & 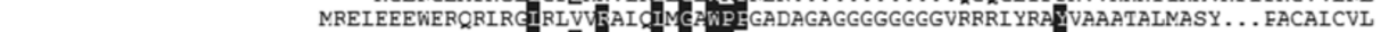 & 73 \\
\hline ImigoR 4 & IHI I $\mathrm{CI}$ WEIR $\ldots \ldots \ldots \ldots \ldots$ ASRIF FI YTAYAAALGVWN $\ldots$ TAEGFIAV & 66 \\
\hline AmeloR2a & 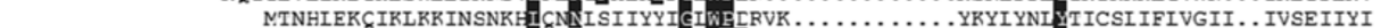 & 66 \\
\hline BmorOR16 & ..... ENFARK AYRIFNNSLCHLF . . . IFFQIIYI & 55 \\
\hline DmeloR43a & ......FGSSWRK EAFVI FVTANN . . . .INQEVYL & 50 \\
\hline DmeloR 59b & .... VIRYVYIFRTCVFFAFGVFY...LFVGFIISY & 69 \\
\hline TcasOR 47 & ...... NFKIYRVYTHFVIINNYLY... NITSIINM & 52 \\
\hline TCasOR28 & NGIFGMYHRSG. . . . . . . NFTIFEKVIAYCNFFFFTIFIPIIGSIYFF & 68 \\
\hline \multirow[t]{2}{*}{ Phumor 4} & .......NRFEC्रIIESIFSFISYFSITSLIINVVIIV & 62 \\
\hline & III & \\
\hline ImiqOR1 & HYTHSETYYRIVDEINLI ILRC्रRFYCEGDAELTEALCXTAY & 180 \\
\hline ImigoR2 & CGLF DEEIE. ETAHCSYICIVCFGCIVRAVIFVNEGITVREIVHIICCAT . . . RAEYGSZEGSLEIRSGYQ & 153 \\
\hline ImigOR3 & AGTRALIC. . VIAVIVGVTSSYVGAIFKMFTICYKRS & 167 \\
\hline ImigoR4 & 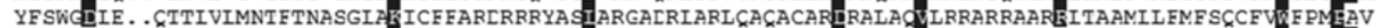 & 162 \\
\hline AmeloR2a & 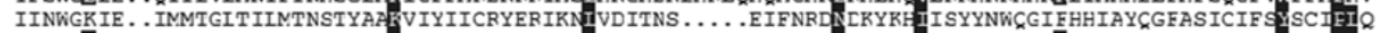 & 157 \\
\hline BmorOR16 & 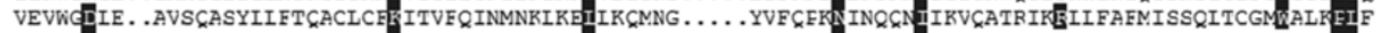 & 146 \\
\hline DmeloR43a & 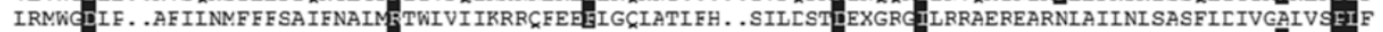 & 144 \\
\hline DmeloR59b & VQEFFN FT PGEFLTSIQVCINVYGASVRSTITYIFLWRIRKIEIIILS.... . ILKRIANLSLRER HHNNVA & 163 \\
\hline TcasOR47 & 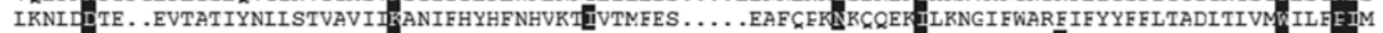 & 143 \\
\hline TcasOR28 & 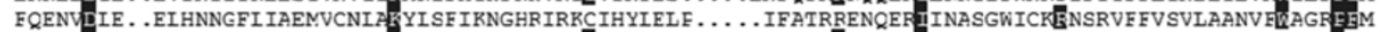 & 159 \\
\hline Phumor 4 & 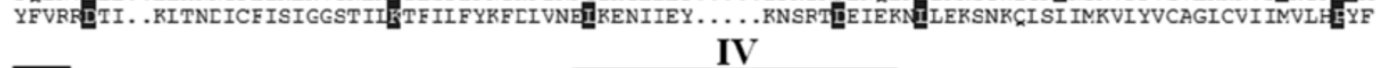 & 153 \\
\hline ImigOR1 & IIINIRISRIS. . . . . HEGVGLG & 263 \\
\hline ImigoR2 & CAISLSITVASAVCIDVFEIRIMMNIAAEI QVINENIAZ IIGCRASGSAYGREEE & 248 \\
\hline ImiqOR3 & QVATLVIVAIWVGAIDAFILVIIVYARGCLIRVINCTIIDNG. . . . . ARSLIGT & 249 \\
\hline ImigoR4 & CVAGIWISC्रISYGVDCIFASVNLIAPAC्रIEIIAGRVAPIG. . & 238 \\
\hline AmeloR2a & VIIINCINNIAIDTIITGEIIITCG & 237 \\
\hline BmorOR16 & LDVGSRR..F....FFDNWNEVSFERSFH. & 224 \\
\hline DmeloR43a & REERA..........FFGVZIEGVSMTSSP. & 221 \\
\hline DmeloR59b & GREF.........WSVYNEFIIWRLGMG & 238 \\
\hline TcasOR47 & IGERR ....F...FSNAWFEYLYLSGRN & 219 \\
\hline TcasOR28 & 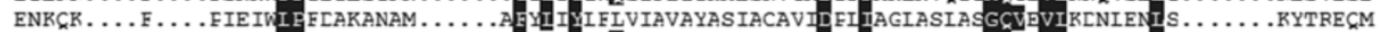 & 236 \\
\hline \multirow[t]{2}{*}{ Phumor 4} & 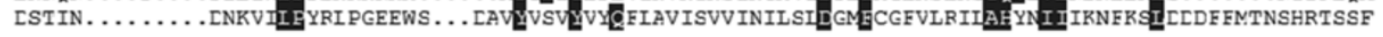 & 239 \\
\hline & & \\
\hline ImiqOR1 & 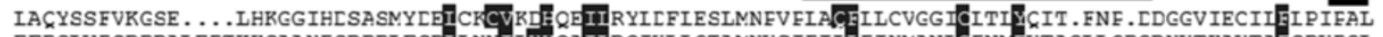 & 355 \\
\hline ImigoR2 & 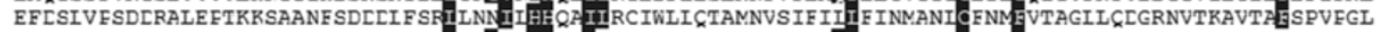 & 346 \\
\hline ImigoR3 & 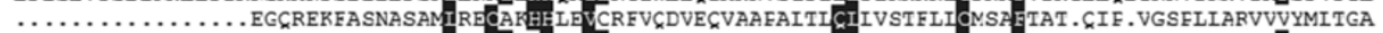 & 328 \\
\hline ImiqOR4 & 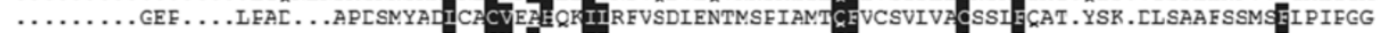 & 318 \\
\hline AmeloR2a & SSIIIERCIARNNNINIERSPSRIYNRFYENIKHCVK & 334 \\
\hline BmoroR16 & $\ldots \ldots \ldots \ldots \ldots \ldots$ HRTYTRISRYNRKIII ECVREFQZVISFTELVELTYNSYII GCIVGSVGI IONSIIRII. VVI. WRSVQFFSIICYLSVNI & 303 \\
\hline DmeloR43a & 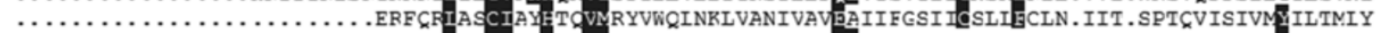 & 291 \\
\hline DmeloR59b & 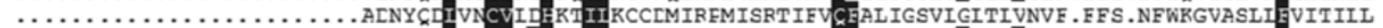 & 309 \\
\hline TcasOR 47 & 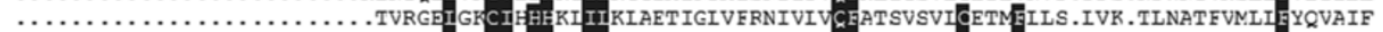 & 289 \\
\hline TCasOR28 & 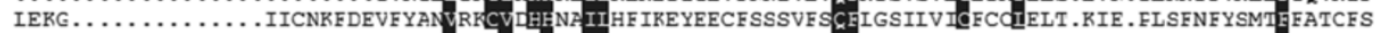 & 318 \\
\hline \multirow[t]{2}{*}{ Phumor 4} & 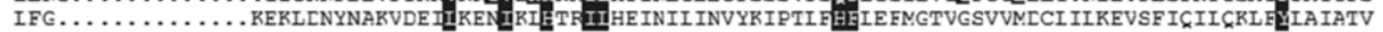 & 323 \\
\hline & VII & \\
\hline ImigOR1 & NRLTHISIINZSYGFYTIIRCNRNIEEENELS & 447 \\
\hline ImigoR2 & ISKEMI ICXVINGTYVIINMI FEVHSLCHI & 436 \\
\hline ImiqOR3 & S.EIIIFCKYCLDVISESERVC्COEI YGSGRSAQGGA FS & 413 \\
\hline ImigOR4 & 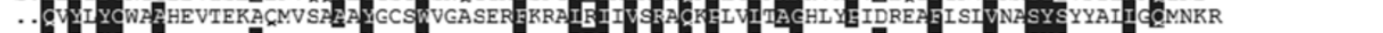 & 403 \\
\hline AmeloR2a & 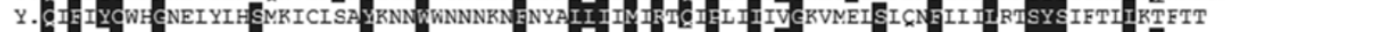 & 419 \\
\hline BmoroR16 & 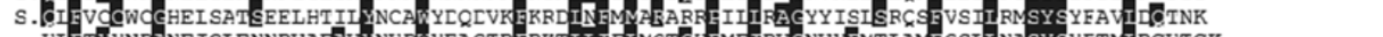 & 388 \\
\hline DmeloR43a & 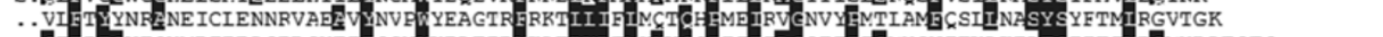 & 376 \\
\hline DmeloR59b & 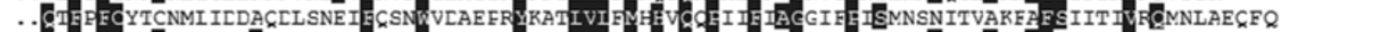 & 398 \\
\hline TcasOR47 & 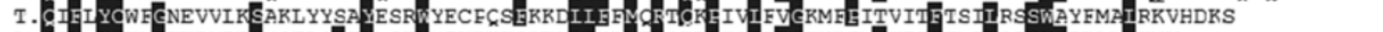 & 376 \\
\hline TcasOR28 & 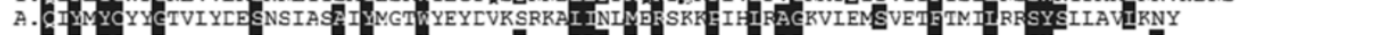 & 401 \\
\hline Phumor 4 & 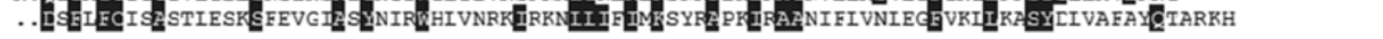 & 409 \\
\hline \multicolumn{3}{|c|}{$\begin{array}{l}\text { Figure } 1 \text { Amino acid sequences alignment of newly identified ORs in locust to odorant receptors from other insects. Residues } \\
\text { conserved in >50\% of the predicted proteins are shaded. The numbers to the right refer to the position of the last residue of each line. } \\
\text { The positions of putative transmembrane domains (I-VII), which were predicted based on LmigOR1, are indicated with black bars. Accession } \\
\text { numbers for the selected odorant receptors: TcasOR28: [GeneBank: EEZ99241]; TcasOR47: [GeneBank: EFA02940]; AmelOR2a: [GeneBank: } \\
\text { XP_003250826]; BmorOR16: [GeneBank: NP_001104832]; PhumOR4: [GeneBank: XP_002427433]; DmelOR43a: [GeneBank: ADK48470]; } \\
\text { DmelOR59a: [GeneBank: AAF47008]. }\end{array}$} \\
\hline
\end{tabular}

Expression of LmigOR1 and LmigOR2 in ORNs in olfactory organs

To determine whether the LmigORs genes were specifically expressed in ORNs, we carried out in situ hybridization using gene-specific probes. We found that only a small subset of the antennal cells present in each section of adult antenna was labelled by the LmigOR 1 antisense probe. We found that more than 10 labelled cells could be detected in each segment (Figure 4a). By contrast, cells labelled by the LmigOR2 antisense probe were found in both antennae and maxillary palps. The number of cells/segment was about 1-3 cells-much less than that 


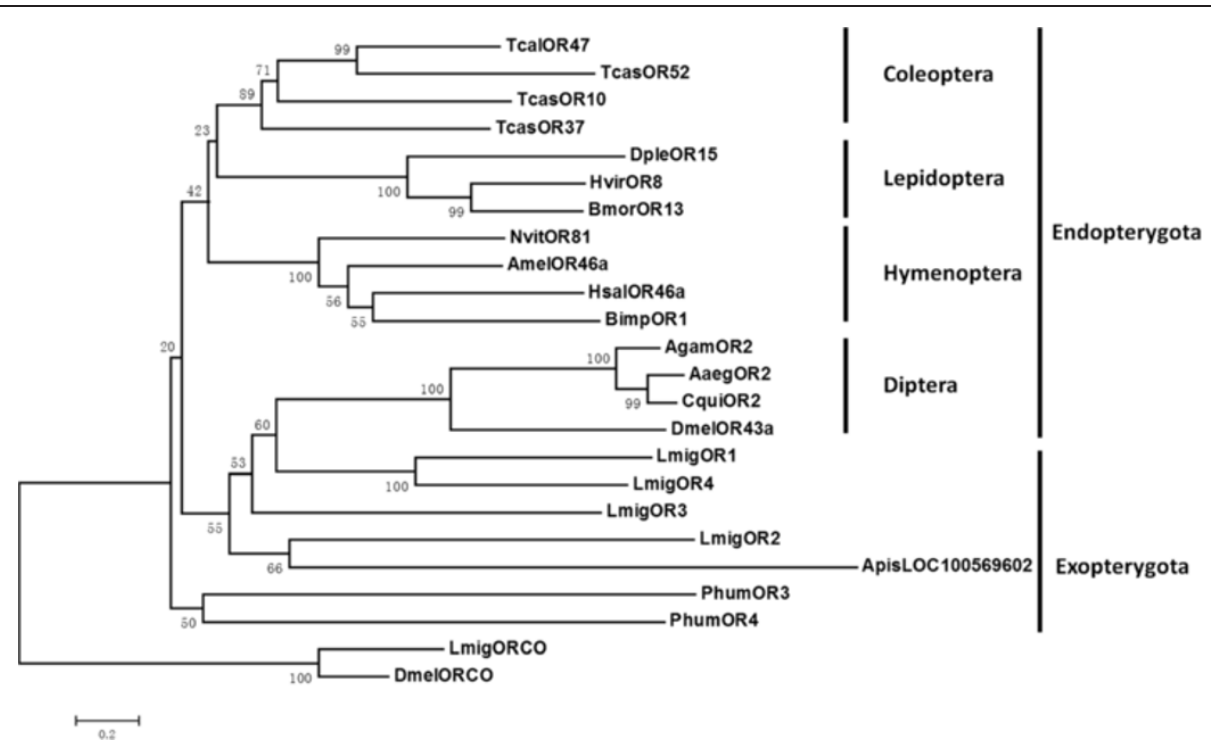

Figure 2 Phylogenetic relationships of LmigORs with odorant receptors from other insects. This distance tree was rooted by declaring the ORco as the outgroup. More closely related insects ORs were removed to facilitate phylogenetic analysis and representation; therefore, many of the lineage-specific subfamily expansions in these insects are not obvious. Accession number for the selected odorant receptors: Hsal46a: [Genebank: EFN79914]; ApisOR46a: [Genebank: XP_003249621]; BimpOR1: [Genebank: XP_003487625]; NvitOR81: [Genebank: NP_001164394]; DpleOR15: [Genebank: EHJ72224]; HvirOR8: [Genebank: CAD31949]; BmorOR13: [Genebank: NP_001166603]; TcasOR37: [Genebank: EEZ99229]; TcasOR10: [Genebank: EFA09294]; TcasOR47: [Genebank: EFA02940]; TcasOR52: [Genebank: EEZ99301]; Dme/OR43a: [Genebank: ADK48356]; AgamOR2: [Genebank: XP_310173]; AaegOR2: [Genebank: XP_001651755]; CquiOR2: [Genebank: XP_001864544]; ApisLOC100569602: [Genebank: XP_003243258]; PhumOR3: [Genebank: XP_002429370]; PhumOR4: [Genebank: XP_002427433]; LmigORco: [Genebank: JN989549]; DmelORco: [Genebank: AAF52031].

for LmigOR1 (Figure $4 \mathrm{~b}-\mathrm{d}$ ). We did not detect any labelled antennal cells expressing LmigOR3 or LmigOR4 (data not shown).

To confirm the neuronal identity of the labelled cells, we performed RNA in situ hybridization on consecutive sections using RNA probes for LmigOR1/2 and LmigORco. The results showed that antennal cells expressing LmigOR $1 / 2$ located within ORNs clusters expressing LmigORco (Figure 5a-d), indicating the putative LmigOR 1 and LmigOR2 expressed in ORNs. This was further verified by two-color in situ hybridization using fluorophore labelled probes (Figure $5 \mathrm{~g}, \mathrm{~h}$ ). We sometimes observed labelling of, not only the cell body, but also the dendritic like structure (Figure 5e, f, h), further identifying these labelled cells as ORNs.

\section{LmigOR1 and LmigOR2 map to distinct subtypes of the basiconic sensilla}

We then carried out an imaging experiment to assign the labelled ORNs to morphologically specific sensillum types. The results demonstrated that some ORNs expressing LmigOR1 and LmigOR2 could be unambiguously attributed to basiconic sensilla (Figure 6a, b). In contrast, we did not observe any neurons labelled with LmigOR1 or LmigOR2 probes in trichoid, coeloconic, or chaetic sensilla. No positive signals could be detected when the sense probe was applied (data not shown). Unlike the colocalization pattern seen with LmigORco, LmigOR1 and LmigOR2 were expressed in discrete subset of ORNs (Figure $6 c-e$ ), indicating they were present in different sensilla subtypes.

\section{Discussion}

We have identified four putative ORs in Locusta migratoria, named as LmigOR1, LmigOR2, LmigOR3 and LmigOR4 and found that they harboured all the characteristic features of insect ORs: seven trans-membrane domains and conserved Ser-Tyr-Ser motif, expression in olfactory organs, and more conserved amino acids at the $\mathrm{C}$ terminus than in the $\mathrm{N}$ terminal region $[2,8,17,31]$. However, the new ORs were highly divergent from known insect ORs. This is consistent with the requirement for recognition of a large number of molecularly diverse odorants, and with species-specific expansion of insect OR gene subfamily lineages. Locust has the special microglomerular systems innervated by branched ORNs and projection neurons and may not follow the law of one receptor to one glomerulus in insect [32]. The complex organization of the olfactory system in locust might increase coding capacity and this was correlated with the complicated behavioural plasticity of the locust. Our analysis of the phylogenetic relationships of locust ORs with ORs from other insects further agreed with the view that orthopteran insects, including the locust, play 


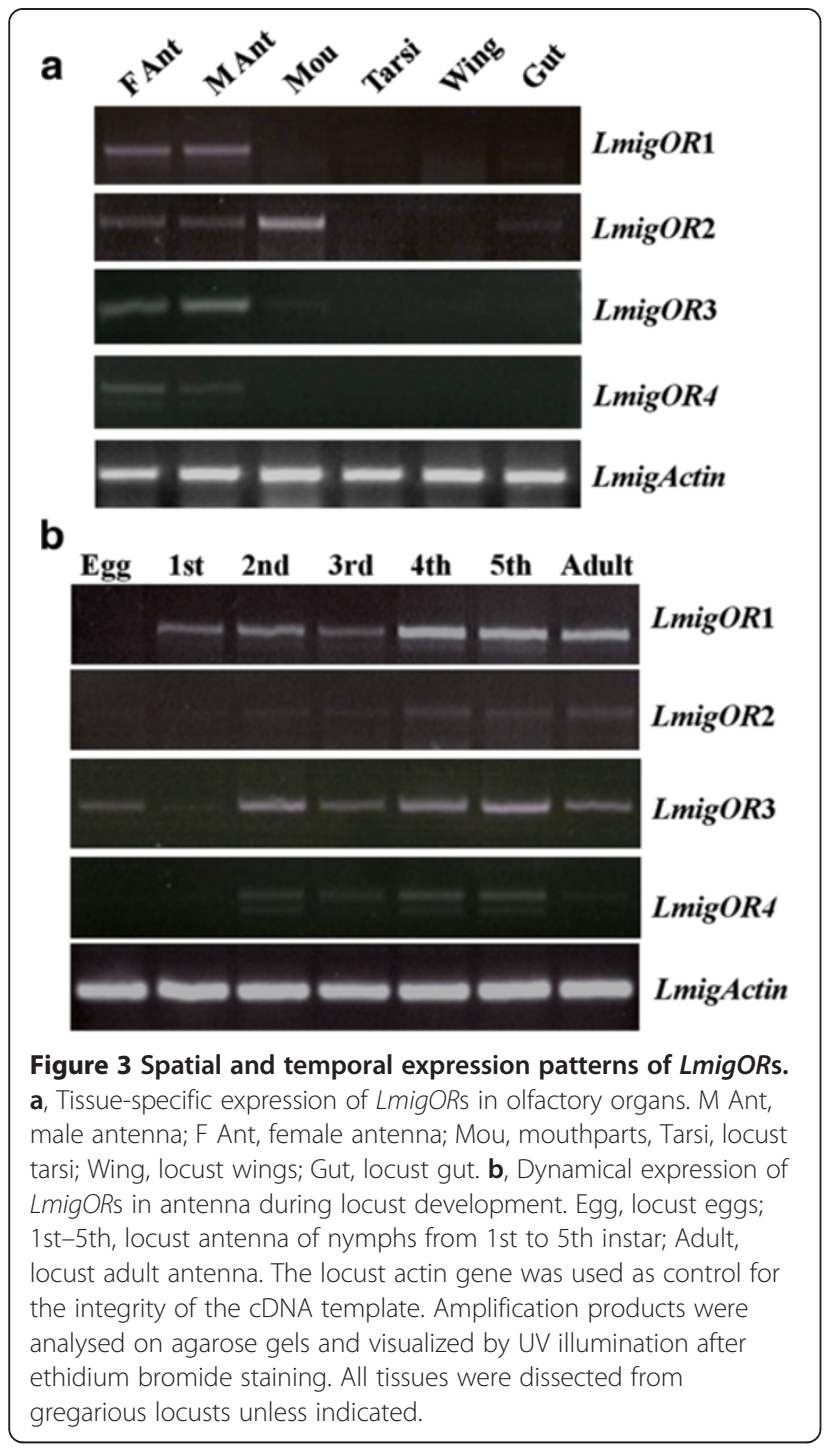

a special role in the study of olfactory evolution, particularly in invertebrates [15].

ORco is generally expressed in insect ORNs and may serve as a marker for ORNs [3,30,33]. Our observation that a few antennal cells expressing LmigOR 1 were located within clusters of LmigORco-expressing cells unambiguously defined them as ORNs. The specific expression of LmigOR1 and LmigOR2 in olfactory organs indicated that they are involved in olfaction. Although both OR transcripts could be detected in antennae, their labelling patterns were different. ORNs expressing individual genes were restricted to distinct subsets of antennal ORNs. The assignment of the two OR genes to different functional types of basiconic sensilla indicate that they have contrasting response profiles. The failure of our attempts to localize the expression of LmigOR3 and LmigOR4 in antenna using RNA in situ hybridization experiments may be partially caused by the high GC content of these ORs, which was $71.6 \%$ and $67 \%$ respectively.

Insects are also equipped with a second nose: the palps. These organs in hawk moth and mosquito serve as detectors for $\mathrm{CO}_{2}[34,35]$, while in Drosophila, they may play a role in taste enhancement [36]. Locust palps have been shown to play critical roles for locating and evaluating food resources [37]. It was therefore not surprising that the expression of LmigOR2 was detected in mouthparts. However, the most fascinating finding was that LmigOR2 was expressed in both antenna and palps. ORs in Drosophila are selectively expressed in only one of these organs $[16,38,39]$. In Drosophila, ORNs housed by basiconic sensilla on antennae and maxillary palpi expressed discrete subsets of OR genes and projected to distinct regions of the antennal lobe, indicating different functions $[39,40]$. In locust, the external morphology of the basiconic sensilla on maxillary palpi resembles that on antennae, except for a prominent socket in connection with a membranous cuticle [28]. Whether the basiconic sensilla on maxillary palpi and antennae have different physiological functions must be experimentally verified. Nevertheless, the overlapping expression of LmigOR2 on antennae and palpi, which resembles the differential expression of some mosquito ORs across all three olfactory appendages, reflected a topographic ordering of sensitivity [34,41].

Some odorant binding proteins, such as LUSH, may serve as triggers that activate ORs through conformational change upon odorant binding [42]. In locust, the odorant-binding protein LmigOBP1 was expressed in all basiconic sensilla on antennae and palps [28,43]. Its coexpression with LmigORs in the same sensilla made it reasonable to guess that these two proteins may interact; this awaits further investigation.

We can only tentatively deduce the functions of LmigORs because of their high sequence divergence from known ORs. Nevertheless, the differential expression of LmigOR1 and LmigOR2 indicates distinct functions. Furthermore, their ubiquitous expression during development and sexindependent expression pattern suggests that these two receptors may be involved in the detection of general odours rather than pheromones. The LmigOR2 is more likely to be involved in feeding because of its abundant expression in mouthparts $[43,44]$.

\section{Conclusions}

We have for the first time identified four novel ORcoding genes in locust, named as LmigOR1, LmigOR2, LmigOR3 and LmigOR4. Their encoded proteins consist of amino acids of 478, 436, 413 and 403; and their theoretical molecular weights were 50.762, 47.867, 44.321 and 44.805 KD respectively. Sequence identities among them ranged from $19.7 \%$ to $35.4 \%$. Our analysis of the phylogenetic relationships of locust ORs with ORs from other 


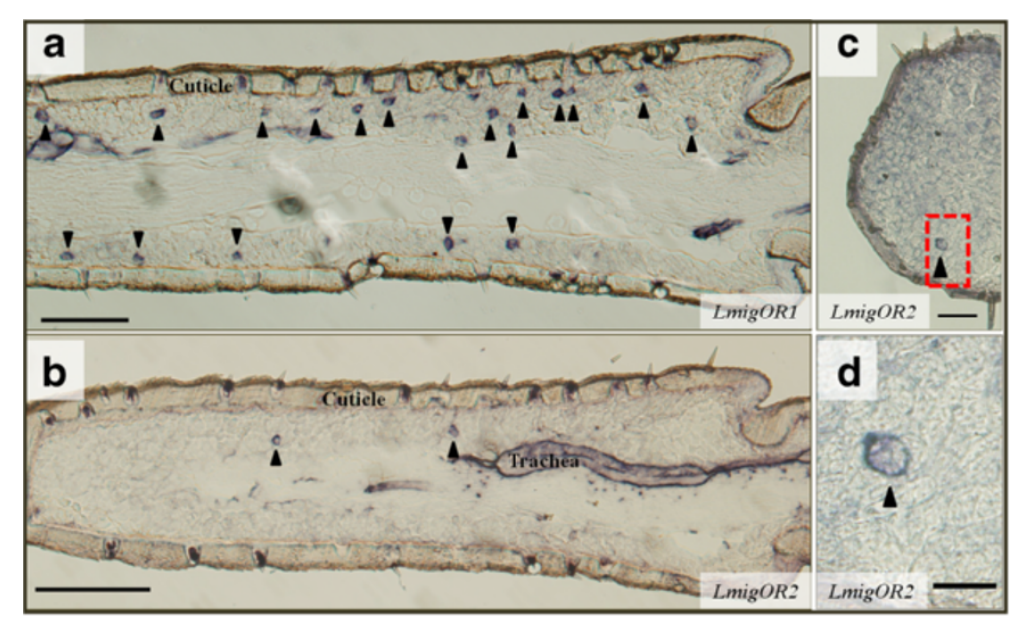

Figure 4 Cellular localization of LmigOR1 and LmigOR2 in olfactory organs. a, Overview of LmigOR1-expressing cells in a locust antennal segment. b, Overview of LmigOR2-expressing cells in a locust antennal segment. c, Cellular localization of LmigOR2 transcripts in maxillary palps. d, A close view of the boxed area in c showing palpal cells expressing LmigOR2. Arrowheads indicate cells expressing LmigOR1 (a) and LmigOR2 (b-d). Scale bars: a, b: $100 \mu \mathrm{m}$; c: $50 \mu \mathrm{m}$; d: $20 \mu \mathrm{m}$.

insects further agreed with the view that orthopteran insects, including the locust, play a special role in the study of olfactory evolution, particularly in invertebrates. They all specifically express in olfaction related organs, antenna or mouthparts in locust. LmigOR1 and LmigOR2 showed differential expression patterns in olfactory organs. LmigOR1 was specifically expressed in antennae, whereas LmigOR2 transcripts could also be detected in mouthparts. Some of the ORNs expressing LmigOR 1 or LmigOR2 could be found in the basiconic sensilla, but the receptors were present in different sensilla subtypes. These results may offer insights into locust olfaction and contribute to the understanding of the evolution of insect chemoreception.

\section{Methods}

Insects

Locusts (L. migratoria) were obtained from the Department of Entomology, China Agricultural University, Beijing, and raised in crowded conditions at $28-30^{\circ} \mathrm{C}$, with $60 \%$ relative humidity, and a light:dark photoperiod of 18:6 h. They were fed daily with fresh wheat shoots. Intact antennae, mouthparts, tarsi, wings, and midguts were dissected using forceps and stored at $-80^{\circ} \mathrm{C}$ until further processing.

\section{cDNA Library construction}

An antennal cDNA library of fourth-instar nymphal locusts was constructed using the SuperScript ${ }^{\oplus}$ Full Length cDNA Library Construction Kit II (Invitrogen, Grand Island, NY, USA) following the manufacturer's protocol. Highly abundant transcripts were subtracted using the genomesaturation hybridization procedure [45]. Sequencing of $\sim 10^{4}$ randomly selected positive clones was performed using an ABI 3730XL capillary sequencer (Invitrogen).

\section{Identification of putative LmigORs-coding genes and} sequence analysis

Vector sequences were detected and masked using Cross_Match. Assembly of clean ESTs into contigs was performed using the Phrap software package (http://www. phrap.org/phredphrapconsed.html). Previously identified insect OR-coding genes were downloaded from NCBI and used as queries to identify putative locust ORs in the formatted EST database by tBlastn searches with the blast-2.2.25+ package (Ftp://ftp.ncbi.nlm.nih.gov/blast/ executables/blast+/2.2.25/). Newly identified ORs were used as query sequences across the database to identify others iteratively. For transmembrane domain predictions, the TMHMM program (v. 2.0) (http://www.cbs.dtu. $\mathrm{dk} /$ services/TMHMM-2.0/) was used. Protein sequence alignment was performed in DNAMAN version 7. An unrooted consensus neighbour-joining tree was calculated using default settings with pairwise gap deletions in MEGA-5 [46]. Branch support was assessed using 1,000 bootstrap replicates.

\section{Rapid amplification of CDNA ends (RACE)}

The gene fragments were extended in both $5^{\prime}$ and $3^{\prime}$ directions for LmigOR1 and 3' directions for LmigOR2 by RACE-PCR with gene-specific primers in conjunction with a SMART-amplified antennal cDNA and SMART adapter-specific primers using the Smarter RACE Kit (Clontech, Palo Alto, CA, USA) according to the manufacturer's manual. Based on the partial LmigORs sequences obtained by blast search of the cDNA library, specific primers for RACE-PCR were designed for touchdown PCRs. PCR products were gel-purified and subcloned using the pGEM-T Easy Kit for sequencing (Promega, Madison, WI, USA). 


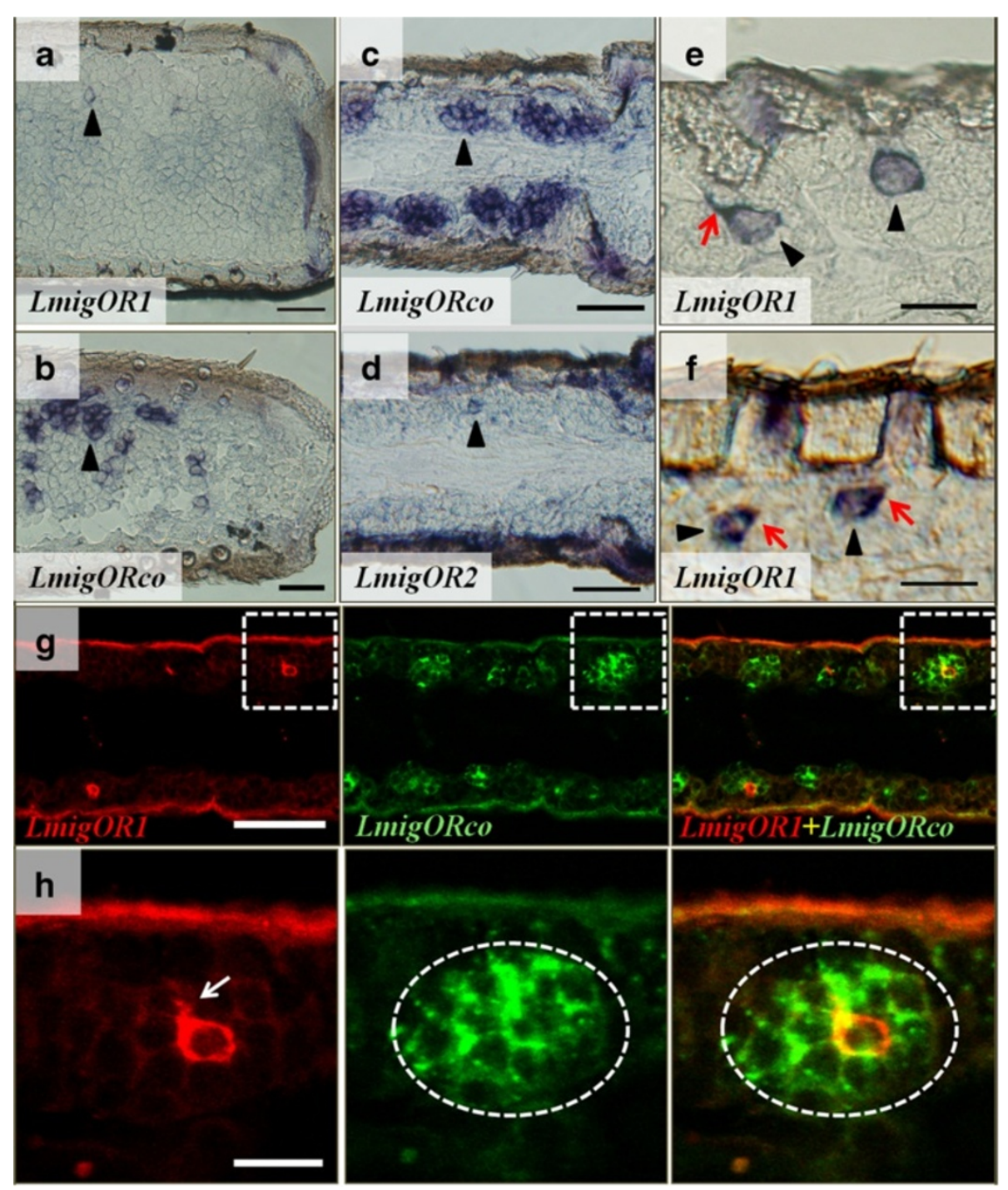

Figure 5 Neuronal identity of antennal cells expressing LmigORs. a-b, The labelling pattern of $L$ migOR1 (a) and LmigORco (b) antisense probe on consecutive sections of locust antenna. $\mathbf{c}-\mathbf{d}$, The labelling pattern of LmigORco (c) and LmigOR2 (d) antisense probe on consecutive sections of locust antenna. e-f, Illustration of occasionally labelled dendritic like structures (indicated by red arrows). $\mathbf{g}$, Two-colour in situ hybridization was performed on longitudinal antennal sections to illustrate the expression of LmigOR1 (Red) and LmigORco (Green). Localization of LmigOR1 expressing cells in cell clusters expressing LmigORco confirmed its neural identity. $\mathbf{h}$, Close view of boxed areas in $\mathbf{g}$. Occasionally labelled dendritic like structures were indicated by arrow. Circled areas indicate ORNs cluster expressing LmigORco and sharing the same sensillum. Scale bar: a-d, g: $50 \mu \mathrm{m} ; \mathbf{e}, \mathbf{f}, \mathbf{h}: 20 \mu \mathrm{m}$.

\section{Expression of LmigORs in different tissues and developmental stages}

Total RNA was isolated from frozen tissues using Trizol reagent (Invitrogen) following the manufacturer's protocols. Reverse transcription was performed using the Quant cDNA Synthesis Kit (Tiangen, Beijing, China) with $1 \mu \mathrm{g}$ unpurified total RNA as template. Non-quantitative RTPCRs were performed with gene-specific primers. To test the integrity of the cDNA preparation, primers for the L. migratoria actin gene [Genebank: AY344445] were used. PCR products were run on $1.2 \%$ agarose gels and visualized by ethidium bromide staining.

\section{Probe preparation and in situ hybridization}

Templates of both ORs were generated by standard PCR using gene-specific primer pairs. Digoxigenin (DIG)- or Biotin- labelled antisense and sense probes were generated from linearized recombinant pGem-T Easy plasmids using the T7/SP6 RNA transcription system (Roche, Basel, Switzerland) following recommended protocols. RNA 


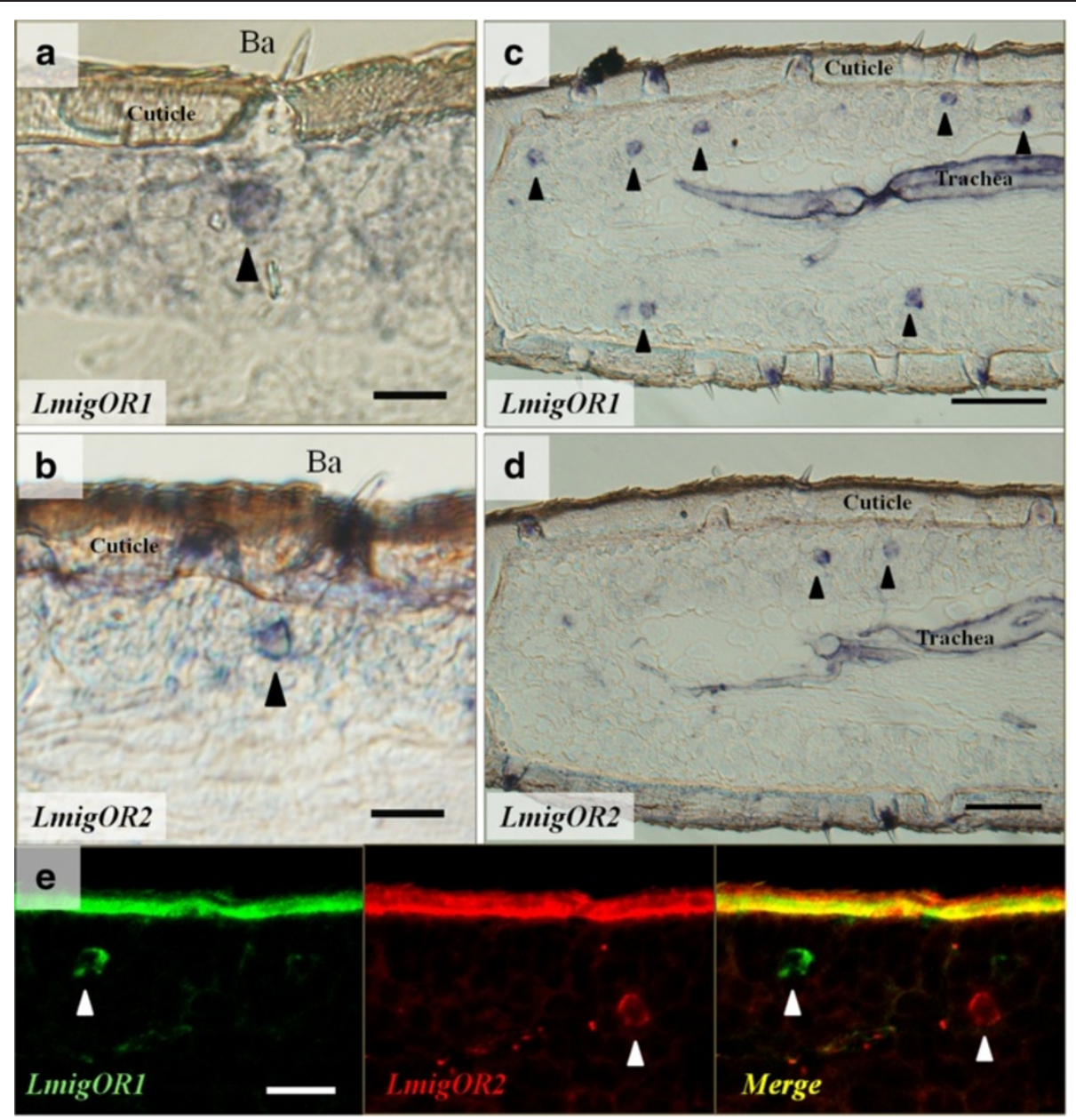

Figure 6 LmigOR1 and LmigOR2 expressed in ORNs housed by basiconic sensilla. a-b, Basiconic sensillum housed ORNs expressing LmigOR1 (a) and LmigOR2 (b). c-e, The expression of LmigOR1 and LmigOR2 in distinct subset of antennal ORNs was verified on consecutive sections (c-d) and by two-color in situ hybridization (e). Fluorescent signals were visualized using detection systems indicating LmigOR1-labelled neurons by green fluorescence and LmigOR2 positive cells by red fuorescence (e). Arrowheads denote antennal cells expressing LmigOR1 (a, $\mathbf{c}$, e) and LmigOR2 (b, d, e). Ba: basiconic sensillum. Scale bar: a, b, e: $20 \mu \mathrm{m}$; c, d: $50 \mu \mathrm{m}$.

probes were subsequently fragmented to an average length of about 300 bp by incubation in carbonate buffer. RNA in situ hybridization was performed according to previously reported procedures [30]. Briefly, antennae were dissected, embedded in the freezing medium (Tissue-Tek O. C.T. Compound; Sakura Finetek Europe, Zoeterwoude, Netherlands). Sections $(12 \mu \mathrm{m})$ were prepared at $-24^{\circ} \mathrm{C}$ using a Jung CM300 cryostat (Leica, Nussloch, Germany) and thaw-mounted on SuperFrost Plus slides (Boster, Wuhan, China). After series of fixing and washing procedures, $100 \mu$ l hybridization solution (Boster) containing RNA probe was placed onto the tissue section. After adding a coverslip, slides were incubated in a humid box at $55^{\circ} \mathrm{C}$ overnight. After hybridization, slides were washed twice for $30 \mathrm{~min}$ in $0.1 \times$ saline-sodium citrate (SSC) at $60^{\circ} \mathrm{C}$, treated with $1 \%$ blocking reagent (Roche) in TBST for $30 \mathrm{~min}$ at room temperature, then incubated for
30 min with an anti-digoxigenin alkaline phosphatase conjugated antibody (Roche,). Hybridization signals were visualized using NBT/BCIP substrate. Tissue sections were analysed on Olympus IX71microscope (Olympus, Tokyo, Japan).

Fluorescent RNA in situ hybridization was carried out in the same way using DIG- and/or biotin-labelled probes. DIG-labelled probes were visualized by the anti-DIG alkaline phosphatase-conjugated antibody in combination with HNPP/Fast Red (Roche). For biotin-labelled probes, TSA kit (Perkin Elmer, MA, USA), including a strepavidin horse radish peroxidase-conjugate and fluorescin-tyramides as substrate, were used. Images were captured on Olympus BX45 confocal microscope and analyzed using FV1000 software. Images were not altered except for adjusting the brightness or contrast uniformly within a single figure (Additional file 1: Table S2). 


\section{Additional file}

Additional file 1: Table S2. Primer sequences used in the present work.

\section{Competing interests}

The authors declare that they have no competing interest.

\section{Authors' contribution}

All authors designed the experiment together. HZX, MG, YY and YWY identified and characterized the expression of odorant receptors. HZX, MG and $Y Y$ performed cellular localization studies, interpreted the results and produced the figures. LZ wrote the paper. All authors read and approved of the final manuscript.

\section{Acknowledgements}

We thank Dr. X. Jin's and two reviewers' comments on the manuscript. This work was supported by grants from the Natural Science Foundation of China (30730012) and the Special Fund for Agro-scientific Research in the Public Interest (200903021).

\section{Author details}

${ }^{1}$ Department of Entomology, China Agricultural University, Beijing 100193, P. R. China. ${ }^{2}$ Current Address: Research Program of Center for DNA Typing, Department of Biochemistry and Molecular Biology, Fourth Military Medical University, Xi'an 710032, P. R. China. ${ }^{3}$ High-tech Research Center, Shandong Academy of Agricultural Sciences, Jinan 250100, China.

Received: 12 March 2013 Accepted: 16 April 2013

Published: 22 April 2013

\section{References}

1. Buck $L$, Axel R: A novel multigene family may encode odorant receptors: a molecular basis for odor recognition. Cell 1991, 65(1):175-187.

2. Vosshall LB, Amrein H, Morozov PS, Rzhetsky A, Axel R: A spatial map of olfactory receptor expression in the Drosophila antenna. Cell 1999, 96(5):725-736.

3. Hallem EA, Ho MG, Carlson JR: The molecular basis of odor coding in the Drosophila antenna. Cell 2004, 117(7):965-979.

4. Hallem EA, Carlson JR: Coding of odors by a receptor repertoire. Cell 2006, 125(1):143-160.

5. Benton R, Sachse S, Michnick SW, Vosshall LB: A typical membrane topology and heteromeric function of Drosophila odorant receptors in vivo. PLOS Biol 2006, 4(2):e20.

6. Lundin C, Käll L, Kreher SA, Kapp K, Sonnhammer EL, Carlson JR, von Heijne G, Nilsson I: Membrane topology of the Drosophila OR83b odorant receptor. FEBS Lett 2007, 581(29):5601-5604.

7. Smart R, Kiely A, Beale M, Vargas E, Carraher C, Kralicek AV, Christie DL, Chen C, Newcomb RD, Warr CG: Drosophila odorant receptors are novel seven transmembrane domain proteins that can signal independently of heterotrimeric G proteins. Insect Biochem Mol Biol 2008, 38(8):770-780.

8. Clyne PJ, Warr CG, Freeman MR, Lessing D, Kim J, Carlson JR: A novel family of divergent seven-transmembrane proteins: candidate odorant receptors in Drosophila. Neuron 1999, 22(2):327-338

9. Gao Q, Chess A: Identification of candidate Drosophila Olfactory receptors from genomic DNA sequence. Genomics 1999, 60(1):31-39.

10. Robertson HM, Warr CG, Carlson JR: Molecular evolution of the insect chemoreceptor gene superfamily in Drosophila melanogaster. PNAS 2003, 100(Suppl 2):14537-14542.

11. Hill CA, Fox AN, Pitts RJ, Kent LB, Tan PL, Chrystal MA, Cravchik A, Collins FH, Robertson HM, Zwiebel LJ: G protein-coupled receptors in Anopheles gambiae. Science 2002, 298(5591):176-178.

12. Robertson HM, Wanner KW: The chemoreceptor superfamily in the honey bee, Apis mellifera: expansion of the odorant, but not gustatory, receptor family. Genome Res 2006, 16(11):1395-1403.

13. Engsontia P, Sanderson AP, Cobb M, Walden KK, Robertson HM, Brown S: The red flour beetle's large nose: an expanded odorant receptor gene family in Tribolium castaneum. Insect Biochem Mol Biol 2008, 38(4):387-397.

14. Krieger J, Klink O, Mohl C, Raming K, Breer H: A candidate olfactory receptor subtype highly conserved across different insect orders. J Comp Physiol 2003, 189(7):519-526.
15. Hansson BS, Stensmyr MC: Evolution of insect olfaction. Neuron 2011, 72(5):698-711.

16. Vosshall $L B$, Wong $A M$, Axel R: An olfactory sensory map in the fly brain. Cell 2000, 102(2):147-159.

17. Krieger J, Raming K, Dewer YME, Bette S, Conzelmann S, Breer H: A divergent gene family encoding candidate olfactory receptors of the moth Heliothis virescens. Eur J Neurosci 2002, 16(4):619-628.

18. Sakurai $T$, Nakagawa $T$, Mitsuno $H$, Mori $H$, Endo $Y$, Tanoue $S$, Yasukochi $Y$, Touhara K, Nishioka T: Identification and functional characterization of a sex pheromone receptor in the silkmoth Bombyx mori. PNAS 2004, 101(47):16653-16658.

19. Wanner K, Anderson A, Trowell S, Theilmann D, Robertson H, Newcomb R: Female-biased expression of odourant receptor genes in the adult antennae of the silkworm, Bombyx mori. Insect Mol Biol 2007, 16(1):107-119.

20. Hallem EA, Carlson JR: The odor coding system of Drosophila. Trends Genet 2004, 20(9):453-459.

21. Cui $X$, Wu C, Zhang L: Electrophysiological response patterns of 16 olfactory neurons from the trichoid sensilla to odorant from fecal volatiles in the locust, Locusta migratoria manilensis. Arch Insect Biochem Physiol 2011, 77(2):45-57.

22. Krieger J, Grosse-Wilde E, Gohl T, Dewer Y, Raming K, Breer H: Genes encoding candidate pheromone receptors in a moth (Heliothis virescens). PNAS 2004, 101(32):11845-11850.

23. Nakagawa T, Sakurai T, Nishioka T, Touhara K: Insect sex-pheromone signals mediated by specific combinations of olfactory receptors. Science 2005, 307(5715):1638-1642.

24. Hassanali A, Njagi PGN, Bashir MO: Chemical ecology of locusts and related acridids. Annu Rev Entomol 2005, 50:223-245

25. Angeli S, Ceron F, Scaloni A, Monti M, Monteforti G, Minnocci A, Petacchi R, Pelosi P: Purification, structural characterization, cloning and immunocytochemical localization of chemoreception proteins from Schistocerca gregaria. Eur J Biochem 1999, 262(3):745-754.

26. Picimbon JF, Dietrich K, Breer H, Krieger J: Chemosensory proteins of Locusta migratoria (Orthoptera: Acrididae). Insect Biochem Mol Biol 2000, 30(3):233-241.

27. Ban L, Scaloni A, Brandazza A, Angeli S, Zhang L, Yan Y, Pelosi P: Chemosensory proteins of Locusta migratoria. Insect Mol Biol 2003, 12(2):125-134.

28. Jin X, Brandazza A, Navarrini A, Ban L, Zhang S, Steinbrecht RA, Zhang L, Pelosi P: Expression and immunolocalisation of odorant-binding and chemosensory proteins in locusts. Cell Mol Life Sci 2005, 62(10):1156-1166.

29. Yu F, Zhang S, Zhang L, Pelosi P: Intriguing similarities between two novel odorant-binding proteins of locusts. Biochem Biophys Res Commun 2009, 385(3):369-374.

30. Yang $Y$, Krieger J, Zhang $L$, Breer $H$ : The olfactory co-receptor Orco from the migratory locust (Locusta migratoria) and the desert locust (Schistocerca gregaria): identification and expression pattern. Int J Biol Sci 2012, 8(2):159-170.

31. Scott K, Brady R Jr, Cravchik A, Morozov P, Rzhetsky A, Zuker C, Axel R: A chemosensory gene family encoding candidate gustatory and olfactory receptors in Drosophila. Cell 2001, 104(5):661-673.

32. Ignell $R$, Anton S, Hansson BS: The Antennal Lobe of Orthoptera - Anatomy and Evolution. Brain Behav Evol 2001, 57(1):1-17.

33. Jones WD, Nguyen TA, Kloss B, Lee KJ, Vosshall LB: Functional conservation of an insect odorant receptor gene across 250 million years of evolution. Curr Biol 2005, 15(4):R119-R121.

34. Lu T, Qiu YT, Wang G, Kwon JY, Rutzler M, Kwon HW, Pitts RJ, van Loon JJ, Takken W, Carlson JR, et al: Odor coding in the maxillary palp of the malaria vector mosquito Anopheles gambiae. Curr Biol 2007, 17(18):1533-1544.

35. Thom C, Guerenstein PG, Mechaber WL, Hildebrand JG: Floral CO2 reveals flower profitability to moths. J Chem Ecol 2004, 30(6):1285-1288.

36. Shiraiwa T: Multimodal chemosensory integration through the maxillary palp in Drosophila. PLoS One 2008, 3(5):e2191.

37. Bernays E, Blaney W, Chapman R: Changes in chemoreceptor sensilla on the maxillary palps of Locusta migratoria in relation to feeding. $J$ Exp Biol 1972, 57(3):745-753.

38. Otaki JM, Yamamoto H: Length analyses of Drosophila odorant receptors. J Theor Biol 2003, 223(1):27-37.

39. Couto A, Alenius M, Dickson BJ: Molecular, anatomical, and functional organization of the Drosophila olfactory system. Curr Biol 2005, 15(17):1535-1547. 
40. Goldman AL, Van der Goes van Naters W, Lessing D, Warr CG, Carlson JR: Coexpression of two functional odor receptors in one neuron. Neuron 2005, 45(5):661-666.

41. latrou K, Biessmann H: Sex-biased expression of odorant receptors in antennae and palps of the African malaria vector Anopheles gambiae. Insect Biochem Mol Biol 2008, 38(2):268-274.

42. Laughlin JD, Ha TS, Jones DN, Smith DP: Activation of pheromone-sensitive neurons is mediated by conformational activation of pheromone-binding protein. Cell 2008, 133(7):1255-1265.

43. Jin $X$, Zhang SG, Zhang L: Expression of odorant-binding and chemosensory proteins and spatial map of chemosensilla on labial palps of Locusta migratoria (Orthoptera: Acrididae). Arthropod Struct Dev 2006, 35(1):47-56.

44. Opstad R, Rogers SM, Behmer ST, Simpson SJ: Behavioural correlates of phenotypic plasticity in mouthpart chemoreceptor numbers in locusts. J Insect Phys 2004, 50(8):725-736.

45. Carninci P: Normalization and subtraction of cap-trapper-selected cdnas to prepare full-length CDNA libraries for rapid discovery of new genes. Genome Res 2000, 10(10):1617-1630.

46. Tamura K, Peterson D, Peterson N, Stecher G, Nei M, Kumar S: MEGA5: molecular evolutionary genetics analysis using maximum likelihood, evolutionary distance, and maximum parsimony methods. Mol Biol Evol 2011, 28(10):2731-2739.

doi:10.1186/1471-2202-14-50

Cite this article as: Xu et al:: Differential expression of two novel

odorant receptors in the locust (Locusta migratoria). BMC Neuroscience 2013 14:50

\section{Submit your next manuscript to BioMed Central and take full advantage of:}

- Convenient online submission

- Thorough peer review

- No space constraints or color figure charges

- Immediate publication on acceptance

- Inclusion in PubMed, CAS, Scopus and Google Scholar

- Research which is freely available for redistribution 